The Annals of Probability

2002, Vol. 30, No. 4, 2081-2107

\title{
THE ABELIAN SANDPILE MODEL ON AN INFINITE TREE
}

\author{
By Christian Maes, Frank Redig and Ellen SaAda
}

\author{
K. U. Leuven, T. U. Eindhoven and C.N.R.S., Rouen
}

\begin{abstract}
We consider the standard Abelian sandpile process on the Bethe lattice. We show the existence of the thermodynamic limit for the finite volume stationary measures and the existence of a unique infinite volume Markov process exhibiting features of self-organized criticality.
\end{abstract}

1. Introduction. Markov processes for spatially extended systems have been around for about 30 years now and interacting particle systems have become a branch of probability theory with an increasing number of connections with the natural and human sciences. While standard techniques and general results have been collected in a number of books such as Liggett (1985), Chen (1992) and Toom (1990) and are capable of treating the infinite volume construction for stochastic systems with locally interacting components, some of the most elementary questions for long range and nonlocal dynamics have remained wide open. We have in mind the class of stochastic interacting systems that during the last decade have invaded the soft condensed matter literature and are sometimes placed under the common denominator of self-organizing systems.

Since the appearance of Bak, Tang and Wiesenfeld (1988), the concept of selforganized criticality (SOC) has excited much interest, and has been applied in a great variety of domains [see, e.g., Turcotte (1999) for an overview]. From the mathematical point of view, the situation is, however, quite unsatisfactory. The models exhibiting SOC are in general very boundary condition dependent [especially the Bak-Tang-Wiesenfeld (BTW) model in dimension 2], which suggests that the definition of an infinite volume dynamics poses a serious problem. Even the existence of a (unique) thermodynamic limit of the finite volume stationary measure is not clear. From the point of view of interacting particle systems no standard theorems are at our disposal. The infinite volume processes we are looking for will be non-Feller and cannot be constructed by monotonicity arguments as in the case of the one-dimensional BTW model [see Maes, Redig, Saada and Van Moffaert (2000)] or the long-range exclusion process [see Liggett (1980)]. On the other hand, to make mathematically exact statements about SOC, it is necessary to have some kind of infinite volume limit, both for statics and for dynamics.

In this paper we continue our study of the BTW model for the case of the Bethe lattice; this is the Abelian sandpile model on an infinite tree. For this system, many

Received December 2000; revised February 2002.

AMS 2000 subject classifications. Primary 82C22; secondary 60K35.

Key words and phrases. Sandpile dynamics, nonlocal interactions, interacting particle systems, thermodynamic limit. 
exact results were obtained in [Dhar and Majumdar (1990)]. In contrast to the one-dimensional case this system has a nontrivial stationary measure. We show here that the finite volume stationary measures converge to a unique measure $\mu$ which is not Dirac and exhibits all the properties of a SOC state. We then turn to the construction of a stationary Markov process starting from this measure $\mu$. The main difficulty to overcome is the strong nonlocality: adding a grain at some lattice site $x$ can influence the configuration far from $x$. In fact the cluster of sites influenced by adding at some fixed site has to be thought of as a critical percolation cluster which is almost surely finite but not of integrable size. The process we construct is intuitively described as follows: at each site $x$ of the Bethe lattice we have an exponential clock which rings at rate $\varphi(x)$. At the ringing of the clock we add a grain at $x$. Depending on the addition rate $\varphi(x)$, we show existence of a stationary Markov process which corresponds to this description. We also extend this stationary dynamics to initial configurations which are typical for a measure $\mu^{\prime}$ that is stochastically below $\mu$.

The paper is organized as follows. In Section 2 we introduce standard results on finite volume Abelian sandpile models and summarize some specific results of [Dhar and Majumdar (1990)] for the Bethe lattice which we need for the infinite volume construction. In Section 3 we present the results on the thermodynamic limit of the finite volume stationary measures and on the existence of infinite volume Markovian dynamics. Section 4 is devoted to proofs and contains some additional remarks.

2. Finite volume Abelian sandpiles. In this section we collect some results on Abelian sandpiles on finite graphs which we will need later on. Most of these results are contained in the review paper by Dhar (1999) or in Ivashkevich and Priezzhev (1998).

2.1. Toppling matrix. Let $V$ denote a finite set of sites. We will always suppose that $V$ is a nearest neighbor connected subset of $\mathbb{Z}^{d}$ or of $\mathbb{T}_{d}$, the infinite homogeneous tree of degree $d+1$. Starting from Section 3 , we specify to the tree.

A matrix $\Delta=\left(\Delta_{x, y}\right)_{x, y \in V}$ indexed by the elements of $V$ is called a toppling matrix if the following hold:

1. for all $x, y \in V, x \neq y, \Delta_{x, y}=\Delta_{y, x} \leq 0$;

2. for all $x \in V, \Delta_{x, x} \geq 1$;

3. for all $x \in V, \sum_{y \in V} \Delta_{x, y} \geq 0$;

4. $\sum_{x, y \in V} \Delta_{x, y}>0$.

The fourth condition ensures that there are sites (so-called dissipative sites) for which the inequality in the third condition is strict. This is fundamental for having a well-defined toppling rule later on. 
Starting from Section 3 of this paper we will choose $\Delta$ to be the lattice Laplacian with open boundary conditions. More explicitly,

$$
\begin{aligned}
& \Delta_{x, x}= \begin{cases}2 d, & \text { if } V \subset \mathbb{Z}^{d}, \\
d+1, & \text { if } V \subset \mathbb{T}_{d},\end{cases} \\
& \Delta_{x, y}=-1 \quad \text { if } x \text { and } y \text { are nearest neighbors. }
\end{aligned}
$$

The dissipative sites then correspond to the boundary sites of $V$. The results on the finite volume Abelian sandpile in this section remain valid for a general toppling matrix $\Delta$.

2.2. Configurations. A height configuration $\eta$ is a mapping from $V$ to $\mathbb{N}=$ $\{1,2, \ldots\}$ assigning to each site a natural number $\eta(x) \geq 1$ ("the number of sand grains" at site $x$ ). A configuration $\eta \in \mathbb{N}^{V}$ is called stable if, for all $x \in V$, $\eta(x) \leq \Delta_{x, x}$. Otherwise $\eta$ is unstable. We denote by $\Omega_{V}$ the set of all stable height configurations. For $\eta \in \mathbb{N}^{V}$ and $V^{\prime} \subset V, \eta_{V^{\prime}}$ denotes the restriction of $\eta$ to $V^{\prime}$.

2.3. Toppling rule. The toppling of a site $x$ corresponding to the toppling matrix $\Delta$ is the mapping

$$
T_{x}: \mathbb{N}^{V} \times V \rightarrow \mathbb{N}^{V}
$$

defined by

$$
T_{x}(\eta)(y)= \begin{cases}\eta(y)-\Delta_{x, y}, & \text { if } \eta(x)>\Delta_{x, x}, \\ \eta(y), & \text { otherwise. }\end{cases}
$$

In words, site $x$ topples if and only if its height is strictly larger than $\Delta_{x, x}$, by transferring $-\Delta_{x, y}$ grains to site $y \neq x$ and losing $\Delta_{x, x}$ grains. Toppling rules commute on unstable configurations. This means, for $x, z \in V$ and $\eta$ such that $\eta(x)>\Delta_{x, x}$ and $\eta(z)>\Delta_{z, z}$,

$$
T_{x} T_{z}(\eta)=T_{z} T_{x}(\eta) .
$$

For $\eta \in \mathbb{N}^{V}$, we say that $\zeta \in \Omega_{V}$ arises from $\eta$ by toppling if there exists an $n$-tuple $\left(x_{1}, \ldots, x_{n}\right)$ of sites in $V$ such that

$$
\zeta=\left(\prod_{i=1}^{n} T_{x_{i}}\right)(\eta) .
$$

The toppling transformation is the mapping

$$
\mathcal{T}: \mathbb{N}^{V} \rightarrow \Omega_{V}
$$

defined by the requirement that $\mathcal{T}(\eta)$ arises from $\eta$ by toppling. The fact that stabilization of an unstable configuration is always possible follows from the existence of dissipative sites (only a finite number of sites have to be toppled a 
finite number of times). Thus only the fact that $\mathcal{T}$ is well defined is less trivial: one has to prove here that for a given unstable configuration every possible stabilization makes the same sites topple the same number of times. Moreover, by (3) the order of the $T_{x_{i}}$ in the product (4) is not important. A complete proof can be found in Meester (2002).

2.4. Addition operators. For $\eta \in \mathbb{N}^{V}$ and $x \in V$, let $\eta^{x}$ denote the configuration obtained from $\eta$ by adding one grain to site $x$, that is, $\eta^{x}(y)=\eta(y)+\delta_{x, y}$. The addition operator defined by

$$
a_{x}: \Omega_{V} \rightarrow \Omega_{V}, \quad \eta \mapsto a_{x} \eta=\mathcal{T}\left(\eta^{x}\right)
$$

represents the effect of adding a grain to the stable configuration $\eta$ and letting a stable configuration arise by toppling. Because $\mathcal{T}$ is well defined, the composition of addition operators is commutative: for all $\eta \in \Omega_{V}, x, y \in V$,

$$
a_{x}\left(a_{y} \eta\right)=a_{y}\left(a_{x} \eta\right) .
$$

2.5. Finite volume dynamics. Let $p$ denote a nondegenerate probability measure on $V$, that is, numbers $p_{x}, 0<p_{x}<1$, with $\sum_{x \in V} p_{x}=1$. We define a discrete time Markov chain $\left\{\eta_{n}: n \geq 0\right\}$ on $\Omega_{V}$ by picking a point $x \in V$ according to $p$ at each discrete time step and applying the addition operator $a_{x}$ to the configuration. This Markov chain has the transition operator

$$
P f(\eta)=\sum_{x \in V} p_{x} f\left(a_{x} \eta\right) .
$$

We can equally define a continuous time Markov process $\left\{\eta_{t}: t \geq 0\right\}$ with infinitesimal generator

$$
L^{\varphi} f(\eta)=\sum_{x \in V} \varphi(x)\left[f\left(a_{x} \eta\right)-f(\eta)\right],
$$

generating a pure jump process on $\Omega_{V}$, with addition rate $\varphi(x)>0$ at site $x$.

2.6. Recurrent configurations, stationary measure. We see here that the Markov chain $\left\{\eta_{n}, n \geq 0\right\}$ has only one recurrent class and its stationary measure is the uniform measure on that class.

Let us call $\mathcal{R}_{V}$ the set of recurrent configurations for $\left\{\eta_{n}: n \geq 0\right\}$, that is, those for which $P_{\eta}\left(\eta_{n}=\eta\right.$ infinitely often $)=1$, where $P_{\eta}$ denotes the distribution of $\left\{\eta_{n}: n \geq 0\right\}$ starting from $\eta_{0}=\eta \in \Omega_{V}$. In the following proposition we list some properties of $\mathcal{R}_{V}$. For the sake of completeness we include the proof which can also be found in Meester (2002).

PROPOSITION 2.1. (i) $\mathcal{R}_{V}$ contains only one recurrent class.

(ii) The addition operators $a_{x}$ generate an Abelian group $G$ of permutations of $\mathcal{R}_{V}$. 
(iii) The group $G$ acts transitively on $\mathcal{R}_{V}$. In particular $|G|=\left|\mathcal{R}_{V}\right|$.

(iv) $\left|\mathcal{R}_{V}\right|=\operatorname{det} \Delta$.

PROOF. (i) We write $\eta \hookrightarrow \zeta$ if in the Markov chain $\zeta$ can be reached from $\eta$ with positive probability. Since sand is added with positive probability on all sites $\left(p_{x}>0\right)$, the maximal configuration $\eta_{\max }$ defined by

$$
\eta_{\max }(x)=\Delta_{x, x}
$$

can be reached from any other configuration. Hence, if $\eta \in \mathcal{R}_{V}$, then $\eta \hookrightarrow \eta_{\max }$; therefore $\eta_{\max } \in \mathcal{R}_{V}$ and $\eta_{\max } \hookrightarrow \eta$ [see, e.g., Chung (1960), page 19].

(ii) Fix $\eta \in \mathcal{R}_{V}$; then there exist $n_{y} \geq 1$ such that

$$
\prod_{y \in V} a_{y}^{n_{y}} \eta=\eta
$$

and

$$
g_{x}=a_{x}^{n_{x}-1} \prod_{y \in V, y \neq x} a_{y}^{n_{y}}
$$

satisfies $\left(a_{x} g_{x}\right)(\eta)=\left(g_{x} a_{x}\right)(\eta)=\eta$. The set

$$
\mathcal{R}^{x}=\left\{\zeta \in \mathcal{R}_{V}:\left(a_{x} g_{x}\right)(\zeta)=\zeta\right\}
$$

is closed under the action of $a_{x}$, contains $\eta$, hence also $\eta_{\max }$ : it is a recurrent class. By (i), $\mathcal{R}^{x}=\mathcal{R}_{V}, a_{x} g_{x}$ is the neutral element $e$ and $g_{x}=a_{x}^{-1}$ if we restrict $a_{x}$ to $\mathcal{R}_{V}$.

(iii) Fix $\zeta \in \mathcal{R}_{V}$ and put $\Psi_{\zeta}: G \rightarrow \mathcal{R}_{V} ; g \mapsto g(\zeta)$. As before $\Psi_{\zeta}(G)$ is a recurrent class; hence $\Psi_{\zeta}(G)=\mathcal{R}_{V}$. If for $g, h \in G, \Psi_{\zeta}(g)=\Psi_{\zeta}(h)$, then $g h^{-1}(\zeta)=\zeta$, and by commutativity $g h^{-1}\left(g^{\prime} \zeta\right)=g^{\prime} \zeta$ for any $g^{\prime} \in G$. Therefore $g h^{-1}(\xi)=\xi$ for all $\xi \in \mathcal{R}_{V}$; thus $g=h$. This proves that $\Psi_{\zeta}$ is a bijection from $G$ to $\mathcal{R}_{V}$.

(iv) Adding $\Delta_{x, x}$ particles at a site $x \in V$ makes the site topple, and $-\Delta_{x, y}$ particles are transferred to $y$. This gives

$$
a_{x}^{\Delta_{x, x}}=\prod_{y \neq x} a_{y}^{-\Delta_{x, y}}
$$

On $\mathcal{R}_{V}$ the $a_{x}$ can be inverted and we obtain the closure relation

$$
\prod_{y \in V} a_{y}^{\Delta_{x, y}}=e
$$

Write

$$
\Delta \mathbb{Z}^{V}=\left\{\Delta n: n=\left(n_{y}\right)_{y \in V} \in \mathbb{Z}^{V}\right\}
$$

where

$$
(\Delta n)_{x}=\sum_{y \in V} \Delta_{x, y} n_{y} .
$$


Consider

$$
\Psi: \mathbb{Z}^{V} \rightarrow G: n \mapsto \prod_{x \in V} a_{x}^{n_{x}}
$$

The map $\Psi$ is a homomorphism from $\mathbb{Z}^{V}$ onto $G$, that is, $\Psi(n+m)=\Psi(n) \Psi(m)$ and $\Psi\left(\mathbb{Z}^{V}\right)=G$. Therefore, $G$ is isomorphic to the quotient $\mathbb{Z}^{V} / \operatorname{Ker}(\Psi)$. By (8), $\Delta \mathbb{Z}^{V}$ is contained in $\operatorname{Ker}(\Psi)$. Conversely, let $n \in \operatorname{Ker}(\Psi)$, and put $n=n^{+}-n^{-}$, where $n_{x}^{+}=\max \left\{n_{x}, 0\right\}, n_{x}^{-}=\max \left\{-n_{x}, 0\right\}$. Since $\Psi(n)=e$, adding to a recurrent $\eta \in \mathcal{R}_{V}$ according to $n^{+}$has the same effect as adding according to $n^{-}$. Therefore, there exist $k^{+}, k^{-} \in\left(\mathbb{Z}^{+}\right)^{V}, \zeta \in \mathcal{R}_{V}$ such that

$$
\eta+n^{+}-\Delta k^{+}=\zeta=\eta+n^{-}-\Delta k^{-}
$$

and we conclude $n \in \Delta \mathbb{Z}^{V}$. This shows that $G$ is isomorphic to $\mathbb{Z}^{V} / \Delta \mathbb{Z}^{V}$ and the latter group has cardinality det $\Delta$.

As a consequence of the group property of $G$, the unique stationary measure is uniform on $\mathcal{R}_{V}$.

\section{COROLlaRY 2.1. (i) The measure}

$$
\mu_{V}=\sum_{\eta \in \mathcal{R}_{V}} \frac{1}{\left|\mathcal{R}_{V}\right|} \delta_{\eta}
$$

is invariant under the action of $a_{x}, x \in V\left(\delta_{\eta}\right.$ is the Dirac measure on configuration $\eta$ ).

(ii) $O n L^{2}\left(\mu_{V}\right)$ the adjoint of $a_{x}$ is

$$
a_{x}^{*}=a_{x}^{-1} .
$$

REMARK. This shows that $\mu_{V}$ is invariant under the Markov processes generated by (6) and (7).

2.7. Allowed configurations. Given a configuration $\eta \in \Omega_{V}$, we say that its restriction $\eta_{W}$ to a nonempty subset $W \subset V$ is a forbidden subconfiguration if, for all $x \in W$,

$$
\eta(x) \leq \sum_{y \in W, y \neq x}\left(-\Delta_{x, y}\right)
$$

A configuration $\eta \in \Omega_{V}$ is called allowed if it does not contain a forbidden subconfiguration. We denote by $\mathcal{A}_{V}$ the set of all allowed configurations.

PROPOSITION 2.2.

$$
\mathcal{A}_{V}=\mathcal{R}_{V}
$$


It is easy to see that toppling or adding cannot create a forbidden subconfiguration, which immediately implies $\mathcal{A}_{V} \supset \mathcal{R}_{V}$. For a proof that $\mathcal{A}_{V}=\mathcal{R}_{V}$ using spanning trees see Ivashkevich and Priezzhev (1998); a direct proof can be found in Meester (2002). For a generalization to nonsymmetric toppling matrices, see Speer (1993).

The property of having a forbidden subconfiguration in $W \subset V$ only depends on the heights at sites $x \in W$. Therefore $\eta \in \mathcal{R}_{V}$ implies $\eta_{W} \in \mathcal{R}_{W}$. This "consistency" property enables us to define allowed configurations on infinite sets.

2.8. Expected toppling numbers. For $x, y \in V$ and $\eta \in \Omega_{V}$, let $n_{V}(x, y, \eta)$ denote the number of topplings at site $y \in V$ by adding a grain at $x \in V$, that is, the number of times we have to apply the operator $T_{y}$ to stabilize $\eta^{x}$. Define

$$
G_{V}(x, y)=\int \mu_{V}(d \eta) n_{V}(x, y, \eta) .
$$

Writing down balance between inflow and outflow at site $y$, one obtains [cf. Dhar (1990)]

$$
\sum_{z \in V} \Delta_{x, z} G_{V}(z, y)=\delta_{x, y},
$$

which yields

$$
G_{V}(x, y)=(\Delta)_{x, y}^{-1} .
$$

In the limit $V \uparrow S$ (where $S$ is $\mathbb{Z}^{d}$ or the infinite tree), $G_{V}$ converges to the Green's function of the simple random walk on $S$.

2.9. Some specific results for the tree. When $V_{n}$ is a binary tree of $n$ generations, many explicit results have been obtained in Dhar and Majumdar (1990). We summarize here the results we need for the construction in infinite volume.

1. When adding a grain on a particular site $0 \in V_{n}$ of height 3 , the set of toppled sites is the connected cluster $C_{3}(0, \eta)$ of sites including 0 having height 3 . This cluster is distributed as a random animal (i.e., its distribution only depends on its cardinality, not on its form). Moreover,

$$
\lim _{n \uparrow \infty} \mu_{V_{n}}\left(\left|C_{3}(0, \eta)\right|=k\right) \simeq C k^{-3 / 2}
$$

as $k$ goes to infinity. The notation $\simeq$ means that if we multiply the left-hand side of (13) by $k^{3 / 2}$, then the limit $k \rightarrow \infty$ is some strictly positive constant $C$.

2. When adding a grain on site $x$, the expected number of topplings at site $y$ satisfies

$$
\lim _{n \uparrow \infty} \int \mu_{V_{n}}(d \eta) n_{V_{n}}(x, y, \eta)=G(x, y),
$$


where $G(x, y)$ is the Green's function of the simple random walk on the infinite tree, that is,

$$
G(0, x)=C 2^{-|x|},
$$

and $|x|$ is the "generation number" of $x$ in the tree.

3. The correlations in the finite volume measures $\mu_{V_{k}}$ can be estimated in terms of the eigenvalues of a product of transfer matrices. This formalism is explained in detail in Dhar and Majumdar (1990), Section 5. Let $f, g$ be two local functions whose dependence sets (see below a precise definition) are separated by $n$ generations. To estimate the truncated correlation function

$$
\mu_{V_{k}}(f ; g)=\int f g d \mu_{V_{k}}-\int f d \mu_{V_{k}} \int g d \mu_{V_{k}},
$$

consider the product of matrices

$$
M_{n}^{k}=\prod_{i=1}^{n}\left(\begin{array}{cc}
1+\gamma_{i}^{k, n} & 1+\gamma_{i}^{k, n} \\
1 & 2+\gamma_{i}^{k, n}
\end{array}\right),
$$

where $\gamma_{i}^{k, n} \in[0,1]$. The meaning of $\gamma_{i}^{k, n}$ is explained in Dhar and Majumdar (1990), but we will only use the fact $0 \leq \gamma_{i}^{k, n} \leq 1$ in Lemma 4.1 below. Let $\lambda_{m}^{n, k}$ (resp. $\lambda_{M}^{n, k}$ ) denote the smallest (resp. largest) eigenvalue of $M_{n}^{k}$. Then

$$
\mu_{V_{k}}(f ; g) \leq C(f, g) \frac{\lambda_{m}^{n, k}}{\lambda_{M}^{n, k}} .
$$

For sites $i$ far from the boundary of $V_{k}$, that is, for fixed $i$ and $n$, in the limit $k \rightarrow \infty, \gamma_{i}^{k, n}$ tends to 1 , and the correlations between local functions in the limit $V_{k} \rightarrow S$ are then governed by the maximal and minimal eigenvalues of $M_{n}=$ $\left(\begin{array}{ll}2 & 2 \\ 1 & 3\end{array}\right)^{n}$. We shall need the estimate of a local function with a function living on the boundary of $V_{k}$; therefore we have to use the full expression (17), (18).

\section{Main results.}

3.1. Notation, definitions. From now on, $S$ denotes the infinite rootless binary tree, $V \subset S$ a finite subset of $S ; \Omega_{V}$ is the set of stable configurations in $V$, that is, $\Omega_{V}=\{\eta: V \rightarrow\{1,2,3\}\}$, and the set of all infinite volume stable configurations is $\Omega=\{1,2,3\}^{S}$. The set $\Omega$ is endowed with the product topology, making it into a compact metric space. For $\eta \in \Omega, \eta_{V}$ is its restriction to $V$, and for $\eta, \zeta \in \Omega$, $\eta_{V} \zeta_{V^{c}}$ denotes the configuration whose restriction to $V$ (resp. $V^{c}$ ) coincides with $\eta_{V}$ (resp. $\zeta_{V^{c}}$ ). As in the previous section, $\mathcal{R}_{V} \subset \Omega_{V}$ is the set of all allowed (or recurrent) configurations in $V$, and we define

$$
\mathcal{R}=\left\{\eta \in \Omega: \forall V \subset S \text { finite, } \eta_{V} \in \mathcal{R}_{V}\right\} .
$$


A function $f: \Omega \rightarrow \mathbb{R}$ is local if there is a finite $V \subset S$ such that $\eta_{V}=\zeta_{V}$ implies $f(\eta)=f(\zeta)$. The minimal (in the sense of set ordering) such $V$ is called the dependence set of $f$ and is denoted by $D_{f}$. A local function can be seen as a function on $\Omega_{V}$ for all $V \supset D_{f}$, and every function on $\Omega_{V}$ can be seen as a local function on $\Omega$. The set $\mathcal{L}$ of all local functions is uniformly dense in the set $\mathcal{C}(\Omega)$ of all continuous functions on $\Omega$.

Througout the paper, we use the following notion of limit by inclusion for a function $f$ on the finite subsets of the tree with values in a metric space $(K, d)$ :

Definition 3.1. Let $\delta=\{V \subset S, V$ finite $\}$ and $f: s \rightarrow(K, d)$. Then

$$
\lim _{V \uparrow S} f(V)=\kappa
$$

if, for all $\varepsilon>0$, there exists $V_{0} \in S$ such that, for all $V \supset V_{0}, d(f(V), \kappa)<\varepsilon$.

DEFINITION 3.2. A collection of probability measures $v_{V}$ on $\Omega_{V}$ is a Cauchy net if, for any local $f$ and for any $\varepsilon>0$, there exists $V_{0} \supset D_{f}$ such that, for any $V, V^{\prime} \supset V_{0}$

$$
\left|\int f(\eta) v_{V}(d \eta)-\int f(\eta) v_{V^{\prime}}(d \eta)\right| \leq \varepsilon
$$

A Cauchy net converges to a probability measure $v$ in the following sense: the mapping

$$
\Psi: \mathcal{L} \rightarrow \mathbb{R}, \quad f \mapsto \Psi(f)=\lim _{V \uparrow S} \int f d \nu_{V}
$$

defines a continuous linear functional on $\mathcal{L}$ [hence on $\mathcal{C}(\Omega)$ ] which satisfies $\Psi(f) \geq 0$ for $f \geq 0$ and $\Psi(1)=1$. Thus by the Riesz representation theorem there exists a unique probability measure on $\Omega$ such that $\Psi(f)=\int f d v$. We write $v_{V} \rightarrow v$ and call this $v$ the infinite volume limit of $v_{V}$.

We will also often consider an enumeration of the tree $S,\left\{x_{0}, x_{1}, \ldots, x_{n}, \ldots\right\}$, and put

$$
T_{n}=\left\{x_{0}, \ldots, x_{n}\right\}
$$

\subsection{Thermodynamic limit of stationary measures.}

THEOREM 3.1. The set $\mathcal{R}$ defined in (19) is a perfect set; that is, the following hold:

(i) $\mathcal{R}$ is compact.

(ii) The interior of $\mathcal{R}$ is empty.

(iii) For all $\eta \in \mathcal{R}$ there exists a sequence $\eta_{n} \neq \eta, \eta_{n} \in \mathcal{R}$, converging to $\eta$. 
For $\eta \in \Omega, x \in S$ we denote by $C_{3}(x, \eta)$ the nearest neighbor connected cluster of sites containing the site $x$ and having height 3 .

THEOREM 3.2. The finite volume stationary measures $\mu_{V}$ defined in (10) form a Cauchy net. Their infinite volume limit $\mu$ satisfies the following:

(i) $\mu(\mathcal{R})=1$;

(ii) $\mu$ is invariant under tree automorphisms and mixing;

(iii) $\mu\left(\eta:\left|C_{3}(0, \eta)\right|<\infty\right)=1$;

(iv) $\int\left|C_{3}(0, \eta)\right| \mu(d \eta)=\infty$.

REMARK. Theorem 3.2(iii) above remains true for the set $C_{1}(0, \eta)$, the nearest neighbor connected cluster of sites containing the origin and having height 1 , and probably also for $C_{2}(0, \eta)$ but this we have not been able to prove.

3.3. Infinite volume dynamics. The finite volume addition operators $a_{x, V}$ [cf. (5)] can be extended to $\Omega$ via

$$
a_{x, V}: \Omega \rightarrow \Omega, \quad \eta \mapsto a_{x, V} \eta=\left(a_{x, V} \eta_{V}\right)_{V} \eta_{V^{c}} .
$$

PROPOSITION 3.1. (i) There exists a measurable subset $\Omega^{\prime}$ of $\mathcal{R}$ with $\mu\left(\Omega^{\prime}\right)=1$ on which the limit

$$
\lim _{V \uparrow S} a_{x, V} \eta=a_{x} \eta
$$

exists, and $a_{x} \eta \in \Omega^{\prime}$. More precisely, $\Omega^{\prime}$ is the subset of configurations for which all the clusters $C_{3}(x, \eta)$ are finite.

(ii) The measure $\mu$ of Theorem 3.2 is invariant under the action of $a_{x}$.

(iii) For every $\eta \in \Omega^{\prime}, a_{x}\left(a_{y} \eta\right)=a_{y}\left(a_{x} \eta\right)$, for all $x, y \in S$.

We now construct a Markov process on $\mu$-typical infinite volume configurations which can be described intuitively as follows. Let $\varphi: S \rightarrow(0, \infty)$; this function will be the addition rate function. To each site $x \in S$ we associate a Poisson process $N_{\varphi}^{t, x}$ (for different sites these Poisson processes are mutually independent) with rate $\varphi(x)$. At the event times of $N_{\varphi}^{t, x}$ we "add a grain" at $x$; that is, we apply the addition operator $a_{x}$ to the configuration. Then $L_{V}^{\varphi}$ introduced in (7) generates a pure jump Markov process on $\Omega$. Indeed, this operator is well defined and bounded on any $L^{p}(\mu)$ space by Proposition 3.1, which implies the following.

Proposition 3.2. $L_{V}^{\varphi}$ is the $L^{p}(\mu)$ generator of the stationary Markov process defined by

$$
\exp \left(t L_{V}^{\varphi}\right) f=\int\left(\prod_{x \in V} a_{x}^{N_{\varphi}^{t, x}} f\right) d \mathbb{P}
$$

where $\mathbb{P}$ denotes the joint distribution of the independent Poisson processes $N_{\varphi}^{t, x}$, and $f \in L^{p}(\mu)$. 
The following condition on the addition rate $\varphi$ is crucial in our construction. Remember $|x|$ is the generation number of $x$ :

SUMMABILITY CONDITION.

$$
\sum_{x \in S} \varphi(x) 2^{-|x|}<\infty
$$

In view of (15) the summability condition ensures that the number of topplings at any site $x \in S$ remains finite almost surely in any finite interval of time when grains are added at intensity $\varphi$.

THEOREM 3.3. If $\varphi$ satisfies condition (23), then we have the following:

(i) The semigroups $S_{V}^{\varphi}(t)=\exp \left(t L_{V}^{\varphi}\right)$ converge strongly in $L^{1}(\mu)$ to a semigroup $S_{\varphi}(t)$.

(ii) $S_{\varphi}(t)$ is the $L^{1}(\mu)$ semigroup of a stationary Markov process $\left\{\eta_{t}: t \geq 0\right\}$ on $\Omega$.

(iii) For any $f \in \mathscr{L}$,

$$
\lim _{t \downarrow 0} \frac{S_{\varphi}(t) f-f}{t}=L^{\varphi} f=\sum_{x \in S} \varphi(x)\left[a_{x} f-f\right],
$$

where the limit is taken in $L^{1}(\mu)$.

Remarks. (i) In Proposition 4.1, we show that $S_{\varphi}(t)$ is a strongly continuous function of $\varphi$.

(ii) In Proposition 4.2, we show that condition (23) is in some sense optimal.

THEOREM 3.4. The process $\left\{\eta_{t}: t \geq 0\right\}$ of Theorem 3.3 admits a cadlag version (right-continuous with left limits).

The intuitive description of the process $\left\{\eta_{t}: t \geq 0\right\}$ is actually correct under condition (23); that is, the process has a representation in terms of Poisson processes:

THEOREM 3.5. If $\varphi$ satisfies condition (23), for $\mu \times \mathbb{P}$ almost every $(\eta, \omega)$ the limit

$$
\lim _{V \uparrow S} \prod_{x \in V} a_{x}^{N_{\varphi}^{t, x}(\omega)} \eta=\eta_{t}
$$

exists. The process $\left\{\eta_{t}: t \geq 0\right\}$ is a version of the process of Theorem 3.3; that is, its $L^{1}(\mu)$ semigroup coincides with $S_{\varphi}(t)$. 
Finally, we can slightly generalize Theorem 3.5 to define the dynamics starting from a measure stochastically below $\mu$. For $\eta, \zeta \in \Omega, \eta \leq \zeta$, if for all $x \in S$, $\eta(x) \leq \zeta(x)$. A function $f: \Omega \rightarrow \mathbb{R}$ is monotone if $\eta \leq \zeta$ implies $f(\eta) \leq f(\zeta)$. Two probability measures $\mu$ and $v$ satisfy $\mu \leq v$ if, for all monotone functions, $\int f d \mu \leq \int f d \nu$.

THEOREM 3.6. Let $\mu^{\prime} \leq \mu$. If $\varphi$ satisfies condition (23), for $\mu^{\prime} \times \mathbb{P}$ almost every $(\eta, \omega)$ the limit

$$
\lim _{V \uparrow S} \prod_{x \in V} a_{x}^{N_{\varphi}^{t, x}(\omega)} \eta=\eta_{t}
$$

exists. The process $\left\{\eta_{t}: t \geq 0\right\}$ is Markovian with $\eta_{0}$ distributed according to $\mu^{\prime}$.

REMARK. The last theorem implies that $\eta \equiv 1$ can be taken as initial configuration.

4. Proofs. This section is devoted to the proofs of the results described above. Some of them will be put in a slightly more general framework so that they can be applied to other cases (where $S$ is not a binary tree or where we have other addition operators $a_{x}$ ) as soon as the existence of a thermodynamic limit of the finite volume stationary measures is guaranteed. The essential cause of difficulty is the nonlocality of the addition operators. The essential simplification is the Abelian property.

\subsection{Thermodynamic limit of stationary measures.}

Proof of THEOREM 3.1. (i), (ii) To see that $\mathcal{R}$ has empty interior, notice that if $\eta \in \mathcal{R}$, there do not exist $x, y \in S$ nearest neighbors such that $\eta(x)=\eta(y)=1$ (that way, $\eta_{\{x, y\}}$ would be a forbidden subconfiguration). Finally $\mathcal{R}$ is closed as an intersection of closed sets.

(iii) Let $\eta_{\max }$ be the maximal configuration, $\eta_{\max }(x)=3$ for all $x \in S$. If $\eta_{V} \in$ $\mathcal{R}_{V}$, then $\eta_{V}\left(\eta_{\max }\right)_{V^{c}} \in \mathcal{R}$. Therefore any $\eta \in \mathcal{R}$ containing an infinite number of sites $x$ for which $\eta(x) \neq 3$ has property (iii) of Theorem 3.1. If $\eta \in \mathcal{R}$ contains only a finite number of sites having height 1 or 2 , then we choose a sequence $\Sigma=\left\{x_{n}: n \in \mathbb{N}\right\} \subset\{x \in S: \eta(x)=3$ and $\eta(y)=3$ for any neighbor of $x\}$ such that two elements of $\Sigma$ are never nearest neighbors, and $\left|x_{n}\right|$ is strictly increasing in $n$. We then define $\eta_{n}(x)=\eta(x)$ for $x \in S \backslash\left\{x_{k} \in \Sigma: 0 \leq k \leq n\right\}$ and $\eta_{n}\left(x_{k}\right)=2$ for $x_{k} \in \Sigma, k \geq n+1$. These $\eta_{n}$ belong to $\mathcal{R}$ since they do not contain any forbidden subconfiguration, and $\eta_{n} \rightarrow \eta$.

Proof of Theorem 3.2. We use $T_{n}$ introduced in (20), but with the $x_{i}$ such that $n \leq m$ implies that the generation numbers satisfy $\left|x_{n}\right| \leq\left|x_{m}\right|$. Then we have

$$
\left|x_{n}\right| \simeq \log _{2} n \text {. }
$$


To prove that the probability measures $\mu_{V}$ form a Cauchy net, it is sufficient to show that for any local function $f: \Omega \rightarrow \mathbb{R}$ we have

$$
\sum_{n}\left|\int f d \mu_{T_{n}}-f d \mu_{T_{n+1}}\right|<\infty .
$$

We do it for $f(\eta)=\eta\left(x_{0}\right)$ by giving an upper bound of the difference $\int f d \mu_{T_{n}}$ $\int f d \mu_{T_{n+1}}$ by a truncated correlation function [cf. (16)]. Then we estimate the latter by the transfer matrix method (cf. Section 2.9 , item 3 ). We abbreviate in what follows $\mu_{n}=\mu_{T_{n}}, \mathcal{R}_{n}=\mathcal{R}_{T_{n}}$.

\section{LEMMA 4.1.}

$$
\left|\mu_{n+1}\left[\eta\left(x_{0}\right)\right]-\mu_{n}\left[\eta\left(x_{0}\right)\right]\right| \leq 3 \mu_{n+1}\left[\eta\left(x_{0}\right) ; I\left(\eta\left(x_{n+1}\right)=3\right)\right] .
$$

PROOF. Every $\eta \in \mathcal{R}_{n}$ can be extended to an element of $\mathcal{R}_{n+1}$ by putting $\eta\left(x_{n+1}\right)=3$ : indeed, adding a site with height 3 cannot create a forbidden subconfiguration. Moreover

$$
\left\{\eta_{T_{n}}: \eta \in \mathcal{R}_{n+1}, \eta\left(x_{n+1}\right)=3\right\}=\mathcal{R}_{n} ;
$$

thus

$$
\mu_{n+1}\left[\eta\left(x_{n+1}\right)=3\right]=\frac{\left|\mathcal{R}_{n}\right|}{\left|\mathcal{R}_{n+1}\right|} .
$$

Since, for any $n,\left|\mathcal{R}_{n+1}\right| \leq 3\left|\mathcal{R}_{n}\right|$, we have

$$
\begin{aligned}
\mu_{n}\left(\eta\left(x_{0}\right)\right) & =\sum_{\eta \in \mathcal{R}_{n}} \frac{1}{\left|\mathcal{R}_{n}\right|} \eta\left(x_{0}\right) \\
& =\sum_{\eta \in \mathcal{R}_{n+1}} \frac{1}{\left|\mathcal{R}_{n+1}\right|} \eta\left(x_{0}\right) I\left(\eta\left(x_{n+1}\right)=3\right) \frac{\left|\mathcal{R}_{n+1}\right|}{\left|\mathcal{R}_{n}\right|} \\
& =\sum_{\eta \in \mathcal{R}_{n+1}} \frac{1}{\left|\mathcal{R}_{n+1}\right|} \eta\left(x_{0}\right) I\left(\eta\left(x_{n+1}\right)=3\right) \frac{1}{\mu_{n+1}\left[\eta\left(x_{n+1}\right)=3\right]} .
\end{aligned}
$$

Therefore

$$
\left|\mu_{n+1}\left(\eta\left(x_{0}\right)\right)-\mu_{n}\left(\eta\left(x_{0}\right)\right)\right| \leq \frac{\mu_{n+1}\left[\eta\left(x_{0}\right) ; I\left(\eta\left(x_{n+1}\right)=3\right)\right]}{\mu_{n+1}\left[\eta\left(x_{n+1}\right)=3\right]} .
$$

The lemma follows now from (26).

Recalling Section 2.9, item 3, we have

$$
\mu_{n}\left[\eta\left(x_{0}\right) ; I\left(\eta\left(x_{n}\right)=3\right)\right] \leq C \frac{\lambda_{m}^{\left|x_{n}\right|,\left|x_{n}\right|}}{\lambda_{M}^{\left|x_{n}\right|,\left|x_{n}\right|}} .
$$


LEMMA 4.2.

$$
\sum_{n=1}^{+\infty} \frac{\lambda_{m}^{\left|x_{n}\right|,\left|x_{n}\right|}}{\lambda_{M}^{\left|x_{n}\right|,\left|x_{n}\right|}}<+\infty
$$

PROOF. We abbreviate $\lambda_{m}^{(n)}=\lambda_{m}^{\left|x_{n}\right|,\left|x_{n}\right|}, \lambda_{M}^{(n)}=\lambda_{M}^{\left|x_{n}\right|,\left|x_{n}\right|}, M(n)=M_{\left|x_{n}\right|}^{\left|x_{n}\right|}, \gamma_{i}=$ $\gamma_{i}^{\left|x_{n}\right|,\left|x_{n}\right|}$. In terms of the trace and the determinant of $M(n)$ we have

$$
\begin{aligned}
& \lambda_{M}^{(n)}=\frac{1}{2}\left(\operatorname{Tr}(M(n))+\sqrt{[\operatorname{Tr}(M(n))]^{2}-4 \operatorname{det}(M(n))}\right) \\
& \lambda_{m}^{(n)}=\frac{1}{2}\left(\operatorname{Tr}(M(n))-\sqrt{[\operatorname{Tr}(M(n))]^{2}-4 \operatorname{det}(M(n))}\right) .
\end{aligned}
$$

Therefore,

$$
\lim _{n \uparrow \infty}\left(\frac{\lambda_{m}^{(n)}}{\lambda_{M}^{(n)}}\right)\left(\frac{[\operatorname{Tr}(M(n))]^{2}}{\operatorname{det}(M(n))}\right)=1 .
$$

To prove the lemma we show that [cf. (24)]

$$
\left(\frac{\operatorname{det}(M(n))}{[\operatorname{Tr}(M(n))]^{2}}\right) \leq\left(\frac{4}{9}\right)^{\left|x_{n}\right|}
$$

Use

$$
\operatorname{det}(M(n))=\prod_{i=1}^{\left|x_{n}\right|}\left(1+\gamma_{i}\right)^{2}
$$

and

$$
\begin{aligned}
\operatorname{Tr}(M(n)) & \geq \operatorname{Tr}\left(\prod_{i=1}^{\left|x_{n}\right|}\left(\begin{array}{cc}
1+\gamma_{i} & 0 \\
0 & 2+\gamma_{i}
\end{array}\right)\right) \\
& =\prod_{i=1}^{\left|x_{n}\right|}\left(1+\gamma_{i}\right)+\prod_{i=1}^{\left|x_{n}\right|}\left(2+\gamma_{i}\right),
\end{aligned}
$$

to estimate [for $1 \leq i \leq\left|x_{n}\right|, 2\left(2+\gamma_{i}\right) \geq 3\left(1+\gamma_{i}\right)$ ]

$$
\begin{aligned}
\left(\frac{\operatorname{det}(M(n))}{[\operatorname{Tr}(M(n))]^{2}}\right) & \leq\left(1+2 \prod_{i=1}^{\left|x_{n}\right|}\left[\frac{2+\gamma_{i}}{1+\gamma_{i}}\right]+\prod_{i=1}^{\left|x_{n}\right|}\left[\frac{2+\gamma_{i}}{1+\gamma_{i}}\right]^{2}\right)^{-1} \\
& \leq\left(1+2 \cdot\left(\frac{3}{2}\right)^{\left|x_{n}\right|}+\left(\frac{3}{2}\right)^{2\left|x_{n}\right|}\right)^{-1} \\
& \leq\left(\frac{4}{9}\right)^{\left|x_{n}\right|} .
\end{aligned}
$$


REMARK. Lemmas 4.1 and 4.2 extend to a general local function $f$ with dependence set $D_{f} \subset T_{n}$ containing $N_{0}$ generations. To obtain Lemma 4.1, replace $\eta\left(x_{0}\right)$ by $f(\eta)$ in its proof. For Lemma 4.2, we have to replace $M_{\left|x_{n}\right|}^{\left|x_{n}\right|}$ by $M_{\left|x_{n}\right|}^{\left|x_{n}\right|-N_{0}}$, and in the computation $\left|x_{n}\right|$ by $\left|x_{n}\right|-N_{0}$. Since $N_{0}$ is finite, the convergence in (25) is unaffected.

Note that by the same proof we obtain that for any two local functions $f, g$ whose dependence sets $D_{f}$ and $D_{g}$ are separated by a distance of $n$ generations we have

$$
\mu(f ; g) \leq C(f, g)\left(\frac{4}{9}\right)^{n} .
$$

So far we have proved the fact that the uniform measures on $\mathcal{R}_{n}$ converge to a unique measure $\mu$. Clearly, $\mu$ concentrates on $\mathcal{R}$ because for any given $V_{0} \subset S$, and $V \supset V_{0}$, the restriction $\eta_{V_{0}}$ is an element of $\mathcal{R}_{V_{0}}$ with $\mu_{V}$-probability 1 .

To prove point (ii), let $\pi: S \rightarrow S$ be a tree automorphism; that is, $\pi$ is bijective and $x$ and $y$ are neighbors if and only if $\pi(x)$ and $\pi(y)$ are neighbors. $\pi$ acts on configurations $\eta \in \Omega$ via $\pi(\eta)(x)=\eta\left(\pi^{-1}(x)\right)$. First note that $\mathcal{R}_{V}=$ $\pi^{-1}\left(\mathcal{R}_{\pi(V)}\right)$. Indeed, if for $W \subset V, \eta_{W}$ is a forbidden subconfiguration, then $\pi(\eta)_{\pi(V)}$ is a forbidden subconfiguration of $\pi(\eta)$ and vice-versa. This implies that for the finite volume measures the equality $\mu_{V} \circ \pi=\mu_{\pi(V)}$ holds. Since $\mu_{V}$ is a Cauchy net, the same holds for $\mu_{\pi(V)}$; hence we conclude

$$
\mu=\lim _{V} \mu_{V}=\lim _{V} \mu_{\pi(V)}=\lim _{V} \mu_{V} \circ \pi=\mu \circ \pi .
$$

The mixing property follows from (29).

Points (iii) and (iv) of the theorem follow from the equality

$$
\lim _{k \rightarrow \infty} k^{-3 / 2} \mu\left(\left|C_{3}(0, \eta)\right|=k\right)=c>0,
$$

which in turn follows from (13), the convergence of $\mu_{n}$ to $\mu$ and the fact that, for any $k$ fixed, the indicator $I\left(\left|C_{3}(0, \eta)\right|=k\right)$ is a local function.

\subsection{Infinite volume toppling operators.}

DEFINITION 4.1. Given the finite volume addition operators $a_{x, V}$ [defined in (21)] acting on $\Omega$, we call a configuration $\eta \in \Omega$ normal if for every $x \in S$ there exists a minimal finite set $V_{x}(\eta) \subset S$ such that, for all $V^{\prime} \supset V \supset V_{x}(\eta)$,

$$
a_{x, V^{\prime}} \eta=a_{x, V} \eta
$$

In other words, for a normal $\eta$, outside $V_{x}(\eta)$, no sites are affected by adding a grain at $x$. In our case, when a particle is added at some site $x \in S$, the 
cluster of toppled sites coincides with the cluster $C_{3}(x, \eta)$ of sites having height 3 including $x$; thus

$$
V_{x}(\eta)=C_{3}(x, \eta) \cup \partial_{e} C_{3}(x, \eta),
$$

where $\partial_{e}$ denotes the exterior boundary. Notice that for a normal configuration $\eta$, by definition,

$$
a_{x}(\eta)=\lim _{V \uparrow S} a_{x, V}(\eta)=a_{x, V_{x}(\eta)}(\eta) .
$$

ProOF OF PROPOSITION 3.1. (i) We show that there is a full measure set $\Omega^{\prime}$ of normal configurations. From (13) and Theorem 3.2,

$$
\int I\left(\left|C_{3}(x, \eta)\right|=n\right) d \mu \simeq C n^{-3 / 2} .
$$

Therefore $\mu$ concentrates on the set $\Omega^{\prime}$ of configurations for which all the clusters $C_{3}(x, \eta)$ are finite, hence for which $\eta$ is normal. Since $I\left(\left|C_{3}(x, \eta)\right|=n\right)$ is measurable, $\Omega^{\prime}$ is measurable. Moreover this set $\Omega^{\prime}$ is closed under the action of the addition operators $a_{y}$, since [cf. (30)]

$$
C_{3}\left(x, a_{y} \eta\right) \subset V_{x}(\eta) \cup V_{y}(\eta) .
$$

(ii) Choose $\varepsilon>0$; pick a local function $f$; fix $V_{n} \uparrow S$ and $n_{0}$ such that $n \geq n_{0}$ implies

$$
\mu\left\{\eta \in \Omega: V_{x}(\eta) \not \subset V_{n}\right\} \leq \frac{\varepsilon}{4\|f\|_{\infty}+1} .
$$

This $n_{0}$ exists since $\mu$ concentrates on normal configurations. We estimate

$$
\begin{aligned}
\mid \int f & \left(a_{x} \eta\right) d \mu-f(\eta) d \mu \mid \\
& \leq\left|\int f\left(a_{x, V_{n}} \eta\right) d \mu-\int f(\eta) d \mu\right|+2\|f\|_{\infty} \mu\left\{\eta \in \Omega: V_{x}(\eta) \notin V_{n}\right\} \\
& \leq \lim _{m}\left|\int f\left(a_{x, V_{n}} \eta\right) d \mu_{V_{m}}-\int f(\eta) d \mu_{V_{m}}\right|+\frac{\varepsilon}{2} \\
& \leq \frac{\varepsilon}{2}+2\|f\|_{\infty} \lim _{m} \mu_{V_{m}}\left(a_{x, V_{n}}(\eta) \neq a_{x, V_{m}}(\eta)\right) \\
& =\frac{\varepsilon}{2}+2\|f\|_{\infty}\left(1-\lim _{m} \mu_{V_{m}}\left(V_{x}(\eta) \subset V_{n}\right)\right) \\
& =\frac{\varepsilon}{2}+2\|f\|_{\infty}\left(1-\mu\left(V_{x}(\eta) \subset V_{n}\right)\right) \leq \varepsilon .
\end{aligned}
$$

In the last step we used that the indicator $I\left(V_{x}(\eta) \subset V_{n}\right)$ is a local function. 
(iii) Let $\eta \in \Omega^{\prime}, x, y \in S$ be two different sites and let $V \supset V_{x}(\eta) \cup V_{x}\left(a_{x} \eta\right) \cup$ $V_{x}\left(a_{y} \eta\right)$. Since $a_{x, V}$ and $a_{y, V}$ commute, we have

$$
\begin{aligned}
a_{x}\left(a_{y} \eta\right) & =a_{x}\left(a_{y, V} \eta\right)=a_{x, V}\left(a_{y, V} \eta\right) \\
& =a_{y, V}\left(a_{x, V} \eta\right)=a_{y, V}\left(a_{x} \eta\right)=a_{y}\left(a_{x} \eta\right) .
\end{aligned}
$$

4.3. Infinite volume semigroup. We now turn to the proofs of Theorems 3.3 and 3.4.

DEFinition 4.2. We define the cluster of $\eta \in \Omega$ at $x \in S$ as

$$
\mathcal{C}(x, \eta)=\left\{y \in S: a_{y} \eta(x) \neq \eta(x)\right\}
$$

and put

$$
G_{\mu}(x, y)=\int I(y \in \mathcal{C}(x, \eta)) d \mu(\eta) .
$$

Finally, for $\varphi: S \rightarrow[0, \infty)$, write

$$
\begin{aligned}
\|f\|_{\varphi} & =\sum_{x \in S} \varphi(x) \int \mu(d \eta)\left|f\left(a_{x} \eta\right)-f(\eta)\right|, \\
\mathcal{B}_{\varphi} & =\left\{f: \Omega \rightarrow \mathbb{R}: f \text { bounded, }\|f\|_{\varphi}<\infty\right\} .
\end{aligned}
$$

REMARK. In our case $y \in \mathcal{C}(x, \eta)$ if $y=x$ or if $\eta(y)=3$ and there exists a nearest neighbor path from $y$ to a neighbor of $x$ along sites having height 3 . The estimate for $G_{\mu}(x, y)$ in Lemma 4.4 below is not dependent on the fact that we are on the tree.

\section{LEMMA 4.3. If}

$$
\sum_{x \in S} \varphi(x) G_{\mu}(y, x)<\infty \quad \text { for all } y \in S,
$$

then all local functions are in $\mathcal{B}_{\varphi}$.

Proof. Let $f$ be a local function with dependence set $D_{f}$. Then $f\left(a_{x} \eta\right) \neq$ $f(\eta)$ if for $y \in D_{f}, a_{x} \eta(y) \neq \eta(y)$, that is, $x \in \mathcal{C}(y, \eta)$,

$$
\begin{aligned}
\|f\|_{\varphi} & =\sum_{x \in S} \varphi(x) \int\left|a_{x} f-f\right| d \mu \\
& =\int \sum_{x \in \cup_{y \in D_{f}} \mathcal{C}(y, \eta)} \varphi(x)\left|a_{x} f-f\right| d \mu \\
& \leq 2\|f\|_{\infty} \sum_{x \in S} \varphi(x) \int I\left(x \in \bigcup_{y \in D_{f}} \mathcal{C}(y, \eta)\right) d \mu \\
& \leq 2\|f\|_{\infty} \sum_{y \in D_{f}} \sum_{x \in S} G_{\mu}(y, x) \varphi(x)<\infty .
\end{aligned}
$$


The next lemma provides a link between $G_{\mu}$ and the Green's function for simple random walk on $S$, that is, between conditions (36) and (23).

LEMMA 4.4.

$$
G_{\mu}(x, y) \leq \sum_{z \sim x} G(y, z)=\delta_{x, y}+3 G(x, y),
$$

where $z \sim x$ means that $z$ and $x$ are neighbors.

PROOF. We have to estimate the probability that $a_{x} \eta(y) \neq \eta(y)$. If by adding a grain at $x$ we influence $y$, this can only be achieved by the toppling of one of the nearest neighbor sites of $y$. Since $\mu$ concentrates on normal configurations,

$$
\begin{aligned}
\mu\left(a_{x} \eta(y) \neq \eta(y)\right) & =\lim _{V \uparrow S} \mu\left(a_{x} \eta(y) \neq \eta(y), V_{x}(\eta) \cup V_{y}(\eta) \subset V\right) \\
& =\lim _{V \uparrow S} \lim _{W \uparrow S} \mu_{W}\left(a_{x, V} \eta(y) \neq \eta(y), V_{x}(\eta) \cup V_{y}(\eta) \subset V\right) \\
& =\lim _{V \uparrow S} \lim _{W \uparrow S} \mu_{W}\left(a_{x, W} \eta(y) \neq \eta(y), V_{x}(\eta) \cup V_{y}(\eta) \subset V\right) \\
& \leq \lim _{W \uparrow S} \mu_{W}\left(a_{x, W} \eta(y) \neq \eta(y)\right) \\
& \leq \lim _{W \uparrow S} \mu_{W}\left(\exists z \in W, z \sim y, n_{W}(z, y, \eta) \geq 1\right) \\
& \leq \lim _{W \uparrow S} \sum_{z \sim y} \int d \mu_{W}(\eta) n_{W}(z, y, \eta) \\
& =\sum_{z \sim y} G(z, y),
\end{aligned}
$$

where we used (12), (14), (30) and (32).

The following lemma completes the proof of Theorem 3.3 and shows that $\mathscr{B}_{\varphi}$ is a natural core for the domain of the generator of the infinite volume semigroup.

LEMMA 4.5. (i) For $f \in \mathscr{B}_{\varphi}$ the net

$$
S_{V}^{\varphi}(t) f=\exp \left(t L_{V}^{\varphi}\right) f=\exp \left(t \sum_{x \in V} \varphi(x)\left(a_{x}-I\right)\right) f
$$

converges in $L^{1}(\mu)$ (as $\left.V \uparrow S\right)$ to a function $S_{\varphi}(t) f \in L^{1}(\mu) . f \mapsto S_{\varphi}(t) f$ defines a semigroup on $\mathcal{B}_{\varphi}$ which is a contraction in both $L^{1}(\mu)$ and $\mathscr{B}_{\varphi}$ norms.

(ii) Under condition (36), the semigroup $S_{\varphi}(t)$ corresponds to a unique Markov process on $\Omega$. 
Proof. We denote by $\|f\|$ the $L^{1}(\mu)$-norm of $f$, and we abbreviate $S_{V}(t)=$ $S_{V}^{\varphi}(t), S(t)=S^{\varphi}(t), L_{V}=L_{V}^{\varphi}$.

(i) First note that $S_{V}(t)$ is well defined on $L^{1}(\mu)$ by Proposition 3.2. By the Abelian property [Proposition 3.1(iii)] we can write, for $V \subset V^{\prime} \subset S$,

$$
\left\|S_{V}(t) f-S_{V^{\prime}}(t) f\right\|=\left\|\left(S_{V^{\prime} \backslash V}(t)-I\right) S_{V}(t) f\right\| .
$$

By Proposition 3.2, $S_{V}(t)$ is the semigroup of a stationary Markov process and hence a contraction on $L^{1}(\mu)$. Therefore

$$
\begin{aligned}
\| S_{V}(t) & \left(S_{V^{\prime} \backslash V}(t)-I\right) f \| \\
\leq & \left\|\left(S_{V^{\prime} \backslash V}(t)-I\right) f\right\| \\
& =\left\|\int_{0}^{t} L_{V^{\prime} \backslash V} S_{V^{\prime} \backslash V}(s) f d s\right\| \\
& \leq \int_{0}^{t}\left\|L_{V^{\prime} \backslash V} f\right\| d s \\
\leq & t \sum_{x \in V^{\prime} \backslash V} \varphi(x) \int\left|\left(a_{x}-I\right) f\right| d \mu \rightarrow 0 \quad \text { as } V, V^{\prime} \uparrow S,
\end{aligned}
$$

where the last step follows from $f \in \mathcal{B}_{\varphi}$. Hence $S_{V}(t) f \rightarrow S(t) f$ in $L^{1}(\mu)$. We show that $S(t) f \in \mathscr{B}_{\varphi}$ :

$$
\begin{gathered}
\sum_{x \in S} \varphi(x) \int\left|S(t) f\left(a_{x} \eta\right)-S(t) f(\eta)\right| \mu(d \eta) \\
\leq \sum_{x \in S} \varphi(x) \int S(t)\left|a_{x} f-f\right| d \mu \\
=\int \sum_{x \in S} \varphi(x)\left|a_{x} f-f\right| d \mu=\|f\|_{\varphi} .
\end{gathered}
$$

Thus $S(t)$ is also a contraction for the $\|\cdot\|_{\varphi}$-norm. We finish with the semigroup property:

$$
\begin{aligned}
S(t) S(s) f & =\lim _{V \uparrow S} S_{V}(t)[S(s) f] \\
& =\lim _{V \uparrow S} \lim _{W \uparrow S} S_{V}(t) S_{W}(s) f
\end{aligned}
$$

and

$$
S(t+s) f=\lim _{V \uparrow S} S_{V}(t) S_{V}(s) f .
$$


Then, since $S_{V}(t)$ is a contraction in $L^{1}(\mu)$,

$$
\left\|S_{V}(t) S_{W}(s) f-S_{V}(t) S_{V}(s) f\right\| \leq\left\|S_{W}(s) f-S_{V}(s) f\right\| .
$$

By (39), the right-hand side of (40) goes to zero as $V, W \uparrow S$.

(ii) If condition (36) is met, then $\mathscr{B}_{\varphi}$ contains all local functions by Lemma 4.3. Therefore, by contractivity the semigroup $S(t)$ on $\mathscr{B}_{\varphi}$ uniquely extends to a semigroup of contractions on $L^{1}(\mu)$. Since, by Proposition 3.2, $S_{V}(t)$ is a Markov semigroup, so is $S(t)$, that is, $S(t) 1=1, S(t) f \geq 0$ if $f \geq 0$. Hence, by Kolmogorov's theorem there is a unique Markov process with semigroup $S(t)$.

REMARK. When $\varphi \equiv 1$, condition (36) is equivalent to

$$
\sum_{x \in S} \int \mu(d \eta) I(x \in \mathcal{C}(y, \eta))=\int|\mathcal{C}(y, \eta)| \mu(d \eta)<+\infty
$$

that is, the clusters must be integrable under $\mu$. For models which exhibit "selforganized criticality," $\mathcal{C}(y, \eta)$ is usually a "finite but critical percolation cluster," implying that $\int|\mathcal{C}(y, \eta)| d \mu=\infty$ [cf. Theorem 3.2(iv), because $\mathcal{C}(y, \eta) \supset$ $\left.\partial_{e} C_{3}(y, \eta)\right]$. Therefore this formalism breaks down for addition rate $\varphi \equiv 1$.

The following lemma proves Theorem 3.4.

LEMMA 4.6. Under condition (36), the process $\left\{\eta_{t}: t \geq 0\right\}$ of Theorem 3.3 is almost surely right-continuous, that is,

$$
\mathbb{P}_{\mu}\left[\lim _{t \downarrow 0} d\left(\eta_{t}, \eta_{0}\right) \geq \varepsilon\right]=0,
$$

where $\mathbb{P}_{\mu}$ is its path-space measure, and the distance $d$ is defined below [in (44)].

Proof. Pick a function $\Psi: S \rightarrow(0,1)$ such that

$$
\sum_{x \in S} \Psi(x)=1
$$

and

$$
\sum_{x, y \in S} \varphi(x) G_{\mu}(x, y) \Psi(y)<\infty .
$$

The distance

$$
d(\eta, \zeta)=\sum_{x \in S}|\eta(x)-\zeta(x)| \Psi(x)
$$


generates the product topology. Denote by $\mathbb{E}_{\mu}$ the expectation w.r.t. $\mathbb{P}_{\mu}$. For $f_{y}(\eta)=\eta(y)$,

$$
f_{y}\left(\eta_{t}\right)-f_{y}\left(\eta_{0}\right)=\int_{0}^{t} L^{\varphi} f_{y}\left(\eta_{s}\right) d s+M_{t}^{y}
$$

where $M_{t}^{y}$ is a centered martingale with quadratic variation

$$
\mathbb{E}_{\mu}\left[\left(M_{t}^{y}\right)^{2}\right]=\mathbb{E}_{\mu}\left[\int_{0}^{t}\left(L^{\varphi} f_{y}^{2}\left(\eta_{s}\right)-2 f_{y}\left(\eta_{s}\right) L^{\varphi} f_{y}\left(\eta_{s}\right)\right) d s\right] .
$$

Using stationarity of $\eta_{s}$ and

$$
\int d \mu\left|L^{\varphi} g\right| \leq 2\|g\|_{\infty} \sum_{x \in S} \sum_{y \in D_{g}} \varphi(x) G_{\mu}(y, x),
$$

for a local bounded function on $\Omega$, we obtain from (45)

$$
\mathbb{E}_{\mu}\left[\left(M_{t}^{y}\right)^{2}\right] \leq C t \sum_{x \in S} \varphi(x) G_{\mu}(y, x) .
$$

Now we can estimate

$$
\begin{aligned}
\mathbb{P}_{\mu}[\exists s & \left.\leq t: \sum_{y \in S}\left|\eta_{s}(y)-\eta_{0}(y)\right| \Psi(y) \geq \varepsilon\right] \\
\leq & \mathbb{P}_{\mu}\left[\int_{0}^{t} d s \sum_{y \in S}\left|L^{\varphi} f_{y}\left(\eta_{s}\right)\right| \Psi(y) \geq \frac{\varepsilon}{2}\right] \\
& +\mathbb{P}_{\mu}\left[\sup _{0 \leq s \leq t}\left|\sum_{y \in S} M_{S}^{y} \Psi(y)\right| \geq \frac{\varepsilon}{2}\right] \\
\leq & \left(\frac{12 t}{\varepsilon}\right) \sum_{x, y \in S} \varphi(x) G_{\mu}(y, x) \Psi(y)+\left(\frac{2}{\varepsilon}\right)^{2} \mathbb{E}_{\mu}\left|\sum_{y \in S} M_{t}^{y} \Psi(y)\right|^{2} \\
\leq & \left(\frac{12 t}{\varepsilon}\right) \sum_{x, y \in S} \varphi(x) G_{\mu}(y, x) \Psi(y)+\left(\frac{2}{\varepsilon}\right)^{2} \mathbb{E}_{\mu}\left[\sum_{y \in S}\left(M_{t}^{y}\right)^{2} \Psi(y)\right] \\
\leq & t C_{\varepsilon} \sum_{x, y \in S} \varphi(x) G_{\mu}(y, x) \Psi(y) .
\end{aligned}
$$

Here we used Markov's and Doob's inequalities in the second step and the Cauchy-Schwarz inequality combined with (42) in the third step. The result (41) follows. 
4.4. Poisson representation. In this section we prove Theorems 3.5 and 3.6. Intuitively it is clear from the Abelian property that the process for which we showed existence in the previous subsection can be represented as $\prod_{x \in S} a_{x}^{N_{\varphi}^{t, x}} \eta$, where $N_{\varphi}^{t, x}$ are independent Poisson processes of intensity $\varphi(x)$.

We take $T_{n}$ as in (20). We say that the product $\prod_{x \in S} a_{x}^{n_{x}} \eta$ exists if for every $y \in S$ there exists $N_{y}$ such that, for all $m, n \geq N_{y}$,

$$
\left|\left[\prod_{x \in T_{n}} a_{x}^{n_{x}} \eta\right](y)-\left[\prod_{x \in T_{m}} a_{x}^{n_{x}} \eta\right](y)\right|=0 .
$$

This is equivalent to the convergence of the sequence $\prod_{x \in T_{n}} a_{x}^{n_{x}} \eta$ in the product topology.

LEMMA 4.7. Under condition (36), the product

$$
\prod_{x \in S} a_{x}^{N_{\varphi}^{t, x}} \eta=\tilde{\eta}_{t}
$$

exists for $\mu$-almost every realization of $N_{\varphi}^{t, x}$ and almost every $\eta$. The process $\left\{\tilde{\eta}_{t}: t \geq 0\right\}$ is a version of the Markov process of Lemma 4.5 .

Proof. Choose a realization of $N_{\varphi}^{t, x}$ such that

$$
\sum_{x \in S} N_{\varphi}^{t, x} G_{\mu}(x, y)<\infty
$$

for every $y$. This happens with probability 1 by condition (36). Define, for $\eta \in \Omega^{\prime}$,

$$
\eta_{T_{n}}(t)=\prod_{x \in T_{n}} a_{x}^{N_{\varphi}^{t, x}} \eta .
$$

Under $\mu, \eta_{T_{n}}(t)$ is stationary in $n$ and $t$. We have

$$
\begin{aligned}
& \mu\left[\left|\left(\eta_{T_{n}}(t)\right)(y)-\left(\eta_{T_{n+1}}(t)\right)(y)\right| \geq 1\right] \\
& \quad \leq \int\left|\left[a_{x_{n+1}}^{N_{\varphi}^{t, x_{n+1}}} \eta_{T_{n}}(t)\right](y)-\left(\eta_{T_{n}}(t)\right)(y)\right| \mu(d \eta) \\
& \quad=\int\left|\left[a_{\left.x_{n+1}^{N_{\varphi}}\right)^{t, x_{n+1}}} \eta\right](y)-\eta(y)\right| \mu(d \eta) \\
& \quad \leq \int \sum_{j=1}^{N_{\varphi}^{t, x_{n+1}}}\left|\left[a_{x_{n+1}^{j}}^{j} \eta\right](y)-\left[a_{x_{n+1}}^{j-1} \eta\right](y)\right| \mu(d \eta) \\
& \quad \leq 6 N_{\varphi}^{t, x_{n+1}} G_{\mu}\left(x_{n+1}, y\right) .
\end{aligned}
$$


In the second and last steps we used the invariance of $\mu$ under $a_{x}$. By the BorelCantelli lemma, (46) implies that, for almost every realization of $N_{\varphi}^{t, x}$,

$$
\mu\left[\exists n_{0}: \forall n \geq n_{0}\left(\eta_{T_{n}}(t)\right)(y)=\left(\eta_{T_{n_{0}}}(t)\right)(y)\right]=1 .
$$

This proves $\mu$-a.s. convergence of the product. To see that $\tilde{\eta}_{t}$ is a version of the Markov process with semigroup $S_{\varphi}(t)$, combine Proposition 3.2 with Theorem 3.3(i) to get, for any local function $f$,

$$
\int d \mu\left|\int d \mathbb{P} f\left(\tilde{\eta}_{t}\right)-S_{\varphi}(t) f\right|=0 .
$$

In the preceding argument we used a particular enumeration of the countable set $S$. But changing it gives again a process with semigroup $S_{\varphi}(t)$. Therefore the limiting process will not depend (up to sets of measure zero) on the chosen enumeration of $S$.

Proof of ThEOREM 3.6. For $\eta \in \Omega^{\prime}$ and $y \in S$ we have the relation [remember (47)]

$$
\eta_{V}(t)(y)=\eta(y)+I(y \in V) \sum_{x \in V} N_{\varphi}^{t, x}-\Delta n_{V}^{t}(y),
$$

where $n_{V}^{t}(x)$, an integer valued random variable, is the number of topplings at site $x$ in the time interval $[0, t]$, when sand is added in $V$. For $T_{n}$ defined in (20) we will first prove that $n_{T_{n}}^{t}$ increases $\mu \times \mathbb{P}$ almost surely to an $L^{1}(\mu \times \mathbb{P})$ random variable $n^{t}$, interpreted as the number of topplings in $[0, t]$ when we add grains according to $N_{\varphi}^{t, x}$. By the Abelian property the sequence $n_{T_{k}}^{t}(0)$ is increasing in $k$. The following estimate is similar to (37):

$$
\begin{aligned}
(\mu \times \mathbb{P}) & \left(\left|n_{T_{k}}^{t}(0)-n_{T_{k+1}}^{t}(0)\right| \geq \varepsilon\right) \\
= & (\mu \times \mathbb{P})\left(n_{x_{k+1}}^{t}(0) \geq \varepsilon\right) \\
& \leq \frac{1}{\varepsilon} \int n_{x_{k+1}}^{t}(0) \mu(d \eta) \times \mathbb{P}(d \omega) \\
& \leq \frac{1}{\varepsilon} \lim _{V \uparrow S} \int n_{x_{k+1}}^{t}(0) I\left(V_{x_{k+1}}(\eta) \cup V_{0}(\eta) \subset V\right) \mu(d \eta) \times \mathbb{P}(d \omega) \\
& \leq \frac{1}{\varepsilon} \lim _{W \uparrow S} \int n_{W}^{t}\left(x_{k+1}, 0, \eta\right) \mu_{W}(d \eta) \times \mathbb{P}(d \omega) \\
& \leq \frac{1}{\varepsilon} t \varphi\left(x_{k+1}\right) G\left(0, x_{k+1}\right) .
\end{aligned}
$$

In the fourth line, $n_{W}^{t}\left(x_{k+1}, 0, \eta\right)$ denotes the number of topplings up to time $t$ at site $0 \in W$ by adding grains at site $x_{k+1} \in W$. By the Borel-Cantelli 
lemma, condition (23) implies the a.s. convergence of $n_{T_{k}}^{t}(0)$, and analogously of every $n_{T_{k}}^{t}(x)$. Pick $(\eta, \omega)$ such that $n_{T_{k}}^{t}(\eta, \omega)$ converges, that is, such that $\sup _{k} n_{T_{k}}^{t}(\eta, \omega)=n^{t}(\eta, \omega)$ is finite [indeed, $n_{T_{k}}^{t}(\eta, \omega)$ is an integer]. If $\eta^{\prime} \leq \eta$, then $n_{T_{k}}^{t}\left(\eta^{\prime}, \omega\right) \leq n_{T_{k}}^{t}(\eta, \omega)$ because we can obtain $\eta$ from $\eta^{\prime}$ by adding sand at sites $x \in S$ for which $\eta^{\prime}(x)<\eta(x)$ thereby increasing the number of topplings. We thus conclude that the convergence of $n_{T_{k}}^{t}(\eta, \omega)$ implies the convergence of $n_{T_{k}}^{t}\left(\eta^{\prime}, \omega\right)$ for all $\eta^{\prime} \leq \eta$.

Now let $\mu^{\prime} \leq \mu$ in the FKG sense. There is a coupling $\mathbb{P}_{12}$ of $\mu^{\prime} \times \mathbb{P}$ and $\mu \times \mathbb{P}$ such that

$$
\mathbb{P}_{12}\left(\left(\left(\eta_{1}, \omega_{1}\right),\left(\eta_{2}, \omega_{2}\right)\right): \omega_{1}=\omega_{2}, \eta_{1} \leq \eta_{2}\right)=1
$$

that is, we use the same Poisson events and couple $\mu^{\prime}$ and $\mu$ according to the optimal coupling [see Strassen (1965)]. Then

$$
\begin{aligned}
\left(\mu^{\prime} \times\right. & \mathbb{P})\left(n_{T_{k}}^{t}(\eta, \omega) \rightarrow n^{t}(\eta, \omega)\right) \\
= & \mathbb{P}_{12}\left(n_{T_{k}}^{t}\left(\eta_{1}, \omega_{1}\right) \rightarrow n^{t}\left(\eta_{1}, \omega_{1}\right)\right) \\
= & \mathbb{P}_{12}\left(n_{T_{k}}^{t}\left(\eta_{1}, \omega_{1}\right) \rightarrow n^{t}\left(\eta_{1}, \omega_{1}\right),\right. \\
& \left.\quad n_{T_{k}}^{t}\left(\eta_{2}, \omega_{2}\right) \rightarrow n^{t}\left(\eta_{2}, \omega_{2}\right), \omega_{1}=\omega_{2}, \eta_{1} \leq \eta_{2}\right) \\
\geq & \mathbb{P}_{12}\left(n_{T_{k}}^{t}\left(\eta_{2}, \omega_{2}\right) \rightarrow n^{t}\left(\eta_{2}, \omega_{2}\right)\right) \\
= & (\mu \times \mathbb{P})\left(n_{T_{k}}^{t}(\eta, \omega) \rightarrow n^{t}(\eta, \omega)\right)=1 .
\end{aligned}
$$

This shows the $\mu^{\prime} \times \mathbb{P}$-almost sure convergence of $n_{T_{k}}^{t}$; hence by (48) the product $\prod_{x \in S} a_{x}^{N_{\varphi}^{t, x}(\omega)} \eta^{\prime}$ converges $\mu^{\prime} \times \mathbb{P}$ almost surely.

As a further result we show that the semigroup $S_{\varphi}(t)$ is continuous as a function of the addition rate $\varphi$. We define

$$
\ell_{1}=\left\{\varphi: S \rightarrow[0, \infty):\|\varphi\|=\sum_{x \in S} \varphi(x) G(0, x)<\infty\right\}
$$

It is a complete metric space (as a closed subset of a Banach space) with the following property: If $\varphi_{n} \in \ell_{1}, \varphi_{n} \uparrow \varphi$ (pointwise) and $\varphi \in \ell_{1}$, then $\varphi_{n} \rightarrow \varphi$ in $\ell_{1}$.

Proposition 4.1. The semigroup $S_{\varphi}(t)$ of Theorem 3.3 is a strongly continuous function of $\varphi$; that is, if $\varphi_{n} \rightarrow \varphi$ in $\ell_{1}$, then, for any local function $f$, $S_{\varphi_{n}}(t) f \rightarrow S_{\varphi}(t) f$. 
PROOF. Let $n_{\varphi}^{t}=\lim _{k \rightarrow \infty} n_{T_{k}}^{t}$ be the number of topplings in $[0, t]$ from sand addition at rate $\varphi$. In the proof of Theorem 3.5 we have shown that this random variable is $\mu \times \mathbb{P}$ almost surely well defined and, after taking limits in (48), satisfies

$$
\eta_{t}=\eta_{0}+N_{\varphi}^{t}-\Delta n_{\varphi}^{t},
$$

where $N_{\varphi}^{t}=\lim _{V \uparrow S} \sum_{x \in V} N_{\varphi}^{t, x}$. Note that if $\psi_{1} \leq \psi_{2}$, the Poisson processes $N_{\psi_{1}}^{t}$ and $N_{\psi_{2}}^{t}$ can be coupled in such a way that, for all $x \in S, N_{\psi_{1}}^{t, x} \leq N_{\psi_{2}}^{t, x}$, and hence, by the Abelian property, $n_{\psi_{1}}^{t}(x) \leq n_{\psi_{2}}^{t}(x)$. Consider a coupling of the four Poisson processes $N_{\varphi}^{t}, N_{\varphi \wedge \varphi_{n}}^{t}, N_{\varphi \vee \varphi_{n}}^{t}$ and $N_{\varphi_{n}}^{t}$ under which the inequalities $X_{1}(t) \geq X_{2}(t)$, $X_{2}(t) \leq X_{3}(t), X_{3}(t) \geq X_{4}(t)$ are satisfied with probability 1 . Let $\tilde{\mathbb{P}}$ denote the law of the marginal $\left(X_{1}, X_{4}\right)$. We have, by a reasoning similar to (49),

$$
\begin{aligned}
& \int d \mu\left(\tilde{\mathbb{E}}\left|n_{\varphi_{n}}^{t}(0)-n_{\varphi}^{t}(0)\right|\right) \\
& \leq \int d \mu\left(\tilde{\mathbb{E}}\left(n_{\varphi_{n}}^{t}(0)-n_{\varphi_{n} \wedge \varphi}^{t}(0)\right)+\tilde{\mathbb{E}}\left(n_{\varphi \vee \varphi_{n}}^{t}(0)-n_{\varphi \wedge \varphi_{n}}^{t}(0)\right)\right. \\
& \left.+\tilde{\mathbb{E}}\left(n_{\varphi \vee \varphi_{n}}^{t}(0)-n_{\varphi}^{t}(0)\right)\right) \\
& \leq t \sum_{x \in S}\left|\varphi_{n}(x)-\varphi(x)\right| G(0, x),
\end{aligned}
$$

which tends to zero for $\varphi_{n} \rightarrow \varphi$ in $\ell_{1}$. Now take a local function $f$, and write $\tilde{D}_{f}=D_{f} \cup \partial_{e} D_{f}$,

$$
\begin{aligned}
\left|S_{\varphi_{n}}(t)(f)-S_{\varphi}(t)(f)\right| & \leq \tilde{\mathbb{P}}\left(n_{\varphi_{n}}^{t}(x) \neq n_{\varphi}^{t}(x) \text { for some } x \in \tilde{D_{f}}\right) \\
& \leq \sum_{x \in \tilde{D}_{f}} \tilde{\mathbb{E}}\left|n_{\varphi}^{t}(x)-n_{\varphi_{n}}^{t}(x)\right| .
\end{aligned}
$$

Combining this with (51) concludes the proof.

One might ask whether we can go beyond condition (23), which essentially guarantees that the expected number of topplings stays finite in the addition process. In the following proposition we show that it is impossible to keep integrable toppling numbers and "rate 1" addition. The relation (52) should be regarded as the infinitesimal version of (50), where $\alpha(x)$ replaces the rate $\varphi(x)$. We then show that $\varphi$ has to depend on $x$.

PROPOSITION 4.2. Let $\alpha: S \rightarrow\{0,1\}$ be a stationary and ergodic random field distributed according to $v$. Denote its density by $\int \alpha(0) v(d \eta)=\rho$. Suppose there exists a measurable transformation $T:\{0,1\}^{S} \times \Omega \rightarrow \Omega$ which satisfies the 
following conditions:

(i) The measure $\mu$ of Theorem 3.2 is invariant under $T(\alpha, \cdot)$ for any $\alpha$. (ii)

$$
T(\alpha, \eta)(x)=\eta(x)+\alpha(x)-\Delta n(\alpha, \eta, x),
$$

with $n(\alpha, \eta, \cdot) \in L^{1}(\mu)$ for $v$ almost every $\alpha$.

Then, $\rho=0$.

PROOF. Taking expectation over $\mu$ in (52) gives

$$
\Delta \Psi(\alpha, x)=\alpha(x),
$$

where $\Psi(\alpha, x)=\int n(\alpha, \eta, x) \mu(d \eta)$. By stationarity of $\mu$ and $v, \Psi(\alpha, x)$ is a stationary random field. Let $\left(x_{t}: t \geq 0\right)$ denote a continuous time simple random walk on $S$, starting at 0 . From (53),

$$
\mathbb{E} \Psi\left(\alpha, x_{t}\right)=\Psi(\alpha, 0)+\mathbb{E} \int_{0}^{t} \alpha\left(x_{s}\right) d s .
$$

Divide this last line by $t$ and let $t \uparrow+\infty$. As $v$ is ergodic (making the last term equal to $\rho)$ and as the process $\Psi\left(\alpha, x_{t}\right)$ is stationary, we conclude that $\rho=0$.

\section{REFERENCES}

Bak, P., TAng, K. and Wiesenfeld, K. (1988). Self-organized criticality. Phys. Rev. A $38364-$ 374.

Chen, M. F. (1992). From Markov Chains to Non-Equilibrium Particle Systems. World Scientific, Singapore.

Chung, K. L. (1960). Markov Chains with Stationary Transition Probabilities. Springer, Berlin.

DHAR, D. (1990). Self organised critical state of sandpile automaton models. Phys. Rev. Lett. 64 $1613-1616$.

Dhar, D. (1999). The Abelian sandpiles and related models. Phys. A 263 4-25.

Dhar, D. and Majumdar, S. N. (1990). Abelian sandpile models on the Bethe lattice J. Phys. A $234333-4350$.

IVAshrevich, E. V. and Priezzhev, V. B. (1998). Introduction to the sandpile model. Phys. A 254 97-116.

Liggett, T. M. (1980). The long range exclusion process. Ann. Probab. 8 861-889.

Liggett, T. M. (1985). Interacting Particle Systems. Springer, Berlin.

Maes, C., Redig, F., SAada E. and van Moffaert, A. (2000). On the thermodynamic limit for a one-dimensional sandpile process. Markov Process. Related Fields 6 1-22.

Meester, R., Redig, F. and Znamenski, D. (2002). The Abelian sandpile; a mathematical introduction. Markov Process. Related Fields 7 509-523.

SpeEr, E. (1993). Asymmetric Abelian sandpile models. J. Statist. Phys. 71 61-74.

STRASSEN, V. (1965). The existence of probability measures with given marginals. Ann. Math. Statist. 36 423-439. 
Toom, A. L., Vasilyev, N. B., Stavskaya, O. N., Mityushin, L. G., Kurdyumov, G. L. and PIRogov, S. A. (1990). Discrete local Markov systems. In Stochastic Cellular Systems: Ergodicity, Memory, Morphogenesis (R. L. Dobrushin, V. I. Kryukov and A. L. Toom, eds.) 1-182. Manchester Univ. Press.

Turcotte, D. L. (1999). Self-organized criticality. Rep. Progr. Phys. 62 1377-1429.

C. MAES

INSTITUUT VOOR THEORETISCHE FySICA

K.U. LEUVEN

Celestijnenlaan 200D

B-3001 LEUVEN

BELGIUM

E-MAIL: christian.maes@fys.kuleuven.ac.be
F. REDIG

TECHNISCHE UNIVERSITEIT EINDHOVEN P.O. Box 513

5600 MB EINDHOVEN

THE NETHERLANDS

E-MAIL: f.h.j.redig@tue.nl

\author{
E. SAADA \\ CNRS, UMR 6085 \\ UNIVERSITÉ DE ROUEN \\ 76821 MONT-SAINT-AIGNAN CEDEX \\ FRANCE \\ E-MAIL: Ellen.Saada@univ-rouen.fr
}

